\title{
Efficient Aerodynamic Computation of a Wing Model Considering Body Effect for the Aeroelastic Application
}

\author{
Seung-Jun Lee*, Dong-Kyun Im* and In Lee** \\ Department of Aerospace Engineering \\ Korea Advanced Institute of Science and Technology, Daejeon, Korea
}

\begin{abstract}
The typical aeroelastic analysis for a complex configuration such as a complete aircraft was done using the aerodynamic results of the wing and the structural modes of a complete aircraft; that is, the aerodynamics of a wing of a complete aircraft is assumed to be not much influenced by the body shape. Nevertheless, the body shape can cause a distortion of aerodynamic pressure on the wing surface and it is necessary to investigate the body effect in flutter analysis. In this reseasrch, MGM inverse design method is applied to include the body effect of a wing-body model which disturbs the pressure distribution on the wing surface.
\end{abstract}

Key Word : Body Effect, Inverse Design, Transonic Small Disturbance, Flutter

\section{Introduction}

Aeroelasticity is the field of studying the interaction between the structural dynamics and aerodynamics. It means that the vehicle structure itself may be unstable when the aerodynamics is coupled. Recently, the aeroelastic problems have been extended to the nonlinear aeroelasticity including various structural and aerodynamic nonlinearities. For the aerodynamic nonlinearity, there are several nonlinear effects like aerodynamic heating, flow seperation and shock wave interaction and so on.

There are several methods to solve these nonlinear aeroelastic problems. One of them, CFD(computational fluid dynamics) approach has been spotlighted to solve many different types of fluid dynamic problems. Specifically, The aeroelastic problems in transonic flow region show very complicated phenomenon like transonic dip. In this phenomenon, the aircraft structure which was designed without considering this effect will be shattered in a few second. TSD(transonic small disturbance) theory has been widely used to solve the transonic aeroelastic problem. It assumes inviscid, irrotational, and small disturbance. Even so, it gives very accurate results with high computation efficiency. On the other hand, TSD theory is difficult to include the above-mentioned nonlinearities. This weak point limits the understanding of CFD aeroelasticity only to the simple wing model. The typical aeroelastic analysis for a complex configuration such as a complete aircraft was done using the aerodynamic results of the wing with the structural modes of a complete aircraft; that is, the aerodynamics of a wing of a complete aircraft is assumed to be not much influenced by the body shape. Of course, the aerodynamics of an aircraft body assumed to be same as those of flat plate. Nevertheless, the body shape can cause a distortion of aerodynamic pressure on the wing surface and it is necessary to investigate the body effect in flutter analysis.

* Research Assistant, Aerospace Engineering Dept., KAIST

** Professor, Aerospace Engineering Dept., KAIST

E-mail : inlee@kaist.ac.kr Tel : +82-42-350-3717

Fax : $+82-42-350-3710$ 
Inverse design method is implemented in this research. Inverse design method is mainly used to change and optimize the airfoil and wing shape automatically according to the target pressure of wing surfaces or object function. There are various kinds of method such as MGM(Modified Garahedian-McFadden) method[1], defection correction method[2], traction method with spring analogy, mesh point, shape function, spline, free-form deformation method and so forth. Among them, MGM method assumes that the changes in the pressure or speed between present and target values depend on the changes of geometry, slope, and the second derivatives.

In this reseasrch, MGM inverse design method is applied to include the body effect which disturbs the pressure distribution on the wing surface. First, the steady aerodynamic field is determined by TSD theory. Next, the pressure difference between the original or ongoing and target pressure is calculated. Lastly, the inverse designed shape is used to analyze the aeroelastic stability; namely, the aerodynamic pressure is implemented to the wing structure in the form of the perturbed pressure by body shape. In next sections, TSD theory, MGM method and its validation are explained briefly. Then a wing-body model is selected and tested to analyze the body effect. This research is the fundamental study of the body effect on aeroelastic influence.

\section{Theoretical Backgrounds}

\section{TSD Aerodynamic Theory}

The governing equations of the transonic flow filed can be arranged as TSD equations with invscid, irrotational, and small disturbance assumptions.

$$
\frac{\partial f_{0}}{\partial \tau}+\frac{\partial f_{1}}{\partial x^{*}}+\frac{\partial f_{2}}{\partial y^{*}}+\frac{\partial f_{3}}{\partial z^{*}}=0
$$

Equation (1) shows the conservative form of TSD equation. Equation (1) is transformed into eq. (2) using the time-accurate approximation factorization(AF) algorithm which consists of a time linearization procedure and Newton iteration technique. Engquist-Osher $(\mathrm{E}-\mathrm{O})$ difference operator has been used in AF algorithm to achieve numerical stability when shock waves exist. Non-reflecting far field boundary conditions are used to achieve accurate and efficient calculation. Details can be found in the Ph. D. thesis of Kim[3] and ref. [4].

$$
L_{\xi} L_{\eta} L_{\zeta} \Delta \phi=-R\left(\phi^{*}, \phi^{n}, \phi^{n-1}, \phi^{n-2}\right)
$$

\section{MGM Inverse Design Method}

MGM inverse design method is one of the residual correction methods. It assumes that the changes in the pressure or speed between present and target values depend on the changes of geometry, slope, and the second derivatives, that is, the surface pressure distribution is a function of the surface ordinate $(S)$, the surface slope $(d S / d t)$, and the surface second derivatives $\left(d^{2} S / d t^{2}\right)$.

$$
F_{0} S_{t}+F_{1} S_{x t}+F_{2} S_{x x t}=R
$$

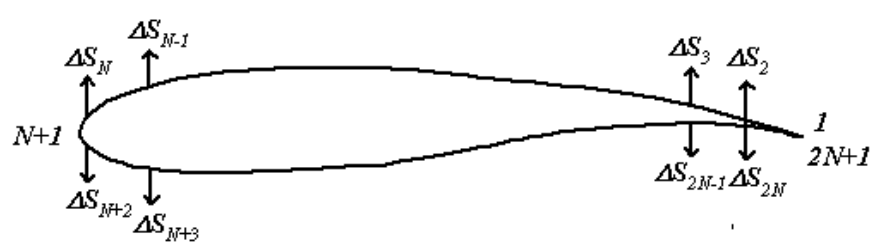

Fig. 1. Numbering of 2-D typical section model 
where $S$ indicates the airfoil geometry, the coefficients $F i(i=0,1,2)$ are constants chosen for a stable iterative process[1], the subscript $t$ and $x$ are time and chordwise position of an airfoil, respectively, and $R$ is the residual, defined as $R=C_{p}^{\text {present }}-C_{p}^{\text {target. }}$, which means the pressure coefficient difference between present and target airfoil.

Equation (3) is corrected to eq. (4) by choosing $\Delta t=1$, upwind difference for the first derivative term, and central differencing for the second derivative term.

$$
A_{i} \Delta S_{i+1}+B_{i} \Delta S_{i}+C_{i} \Delta S_{i-1}=R_{i}
$$

Here, the coefficients $A_{i}, B_{i}, C_{i}$ for upper surface points, are as follows.

$$
\begin{aligned}
& A_{i}=-2 F_{2} /\left(x_{i+1}-x_{i}\right)\left(x_{i+1}-x_{i-1}\right) \\
& B_{i}=F_{0}+F_{1} /\left(x_{i}-x_{i-1}\right)+2 F_{2} /\left(x_{i+1}-x_{i}\right)\left(x_{i}-x_{i-1}\right) \\
& C_{i}=-F_{1} /\left(x_{i}-x_{i-1}\right)-2 F_{2} /\left(x_{i}-x_{i-1}\right)\left(x_{i+1}-x_{i-1}\right) \\
& R_{i}=R_{i}
\end{aligned}
$$

\section{Results}

\section{Validation of MGM Inverse Design Method}

To validate the MGM inverse design method, the recovered 2-D airfoil section shape, pressure and slope data are compared with the target airfoil information. Figure 2 shows the validation results of MGM inverse design method. Figure 2 is the validation of the symmetric airfoils. All tests are done under Mach number 0.7 and angle of attack 0 degree condition. In all figures, a dark line represents the inversed result, a light solid line represents the initial airfoil information and the dotted line or points shows a target airfoil data. In fig. 2(a) the pressure coefficient of upper and lower surface for the inverse designed airfoil coincide with the one for the target airfoil. Figure 2 (b) shows that the inverse designed airfoil shape is showing a good agreement with the target airfoil shape. Figure 2 (c) shows slope data of airfoils. Actually slope data is important for TSD aeroelastic analysis. The grid system of TSD analysis does not vary along the structural motion. Simply the slope information at the center position changes according to the deflection of structure. This advantage makes the iterative inverse design method to be applied easily without regeneration of a new grid system for a new updated airfoil.

\section{Wing-Body Model Details}

Figure 3 is a schematic geometry for NACA RM L51F07 experimental wing-body model[5]. The airfoil is NACA 65A006. The aspect ratio and its taper ratio is 4 and 0.6 respectively. Thereare no incidence angle, dihedral angle, and geometric twist. The wing is swept back by 45 degree, and the fuselage is symmetric along $\mathrm{x}$ axis. The geometry of the model is given as follows.

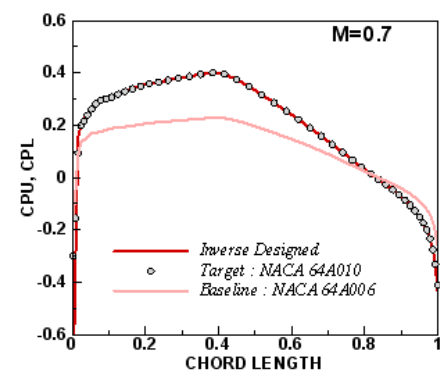

(a) $\mathrm{C}_{\mathrm{p}}$ distribution

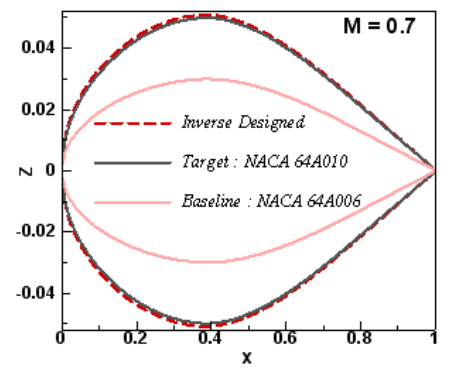

(b) Airfoil shape

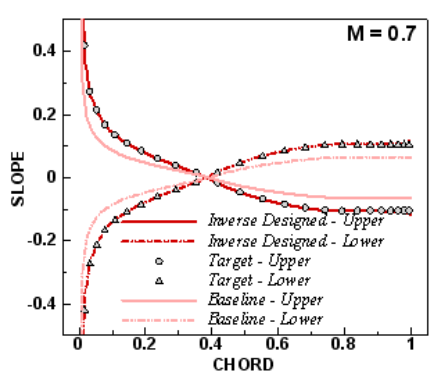

(c) Slope

Fig. 2. Validation of MGM method(baseline:NACA64A006, target:NACA64A010) 


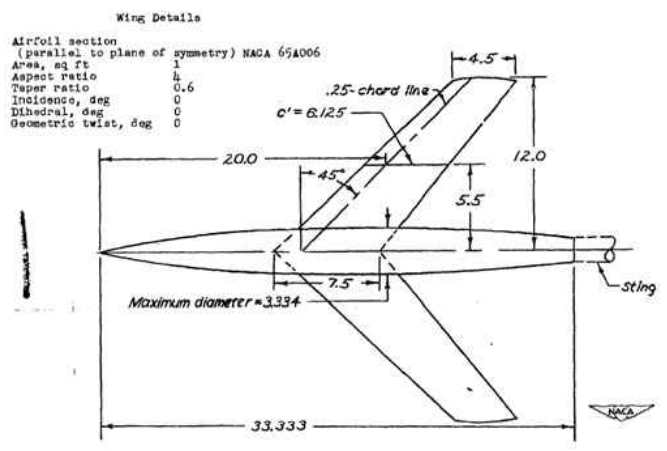

(a) top view

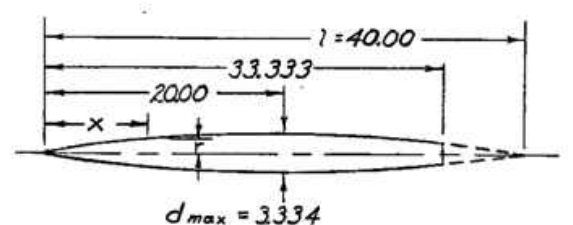

(b) side view

Fig. 3. NACA RM L51F07 wing-body model in inch

\section{Analysis Results}

The wing-body model is analyzed by Euler code. This Euler code is based on the cell-centered finite volume method with 2nd order upwind TVD scheme. The hyperbolic equations are solved by implicit ADI and dual time stepping method[6].

Figure 4 shows the grid for wing-body model. Figure 4 (a) is consisted of $277 \times 45 \times 121$ grid points and fig. 4 (b) shows the convergence history for the grid test. It takes 24 parallel computers and $5830 \mathrm{CPU}$ time. The test condition is Mach number 0.6, 0.8, 0.875, 0.9, 0.93, 0.96 and angle of attack 0, 2, 4 degree according to the experiments of the ref. [5]. In this paper, only the case of Mach number 0.9 and angle of attack 0 degree is presented to analyze the body effect.

The pressure coefficients difference of wing and wing-body model appears on fig. 5. Each plot has TSD result for clean wing model, experimental data from ref. [5], and Euler result for wing-body model. All pressure coefficients are extracted at 20\%, 40\%, 60\%, 80\% position of semi-span. Figure 5 shows that the region near root is more influenced by body and the effect is weak at the wing tip region. As shown in fig. 5 (c), the disturbed pressure coefficients disppear almost at the wing tip region. In 40\% semi-span region, the influenced pressure coefficients is somewhat higher than the other region. It also become weak and disappeare at the near tip region.

Figure 6 shows the pressure coefficient contour plotted. As shown in fig. 5 and 6 , the pressure contour of a wing-body model is slightly increased even if the flight conditions are same. This difference makes the airfoil thick in inverse design point of view. The thicker inverse designed airfoil allows the compressibility change at the wing root on the wing surface like fig. 6 (a). This figure shows only zero angle of attack case. When the angle of attack increases, the compressibility on the wing surface changes a lot. Even though the effect of Mach number and angle of attack caused by fuselage are not presented in this paper, those parameter variation can cause a significant change on surface pressure distribution.

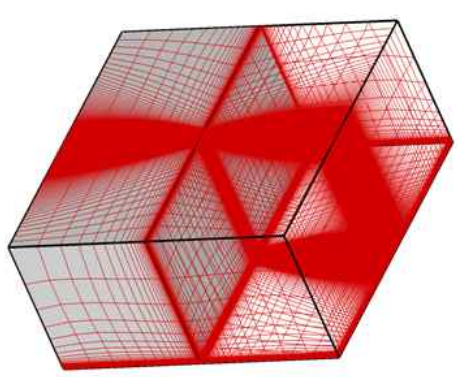

(a) Wing-body model

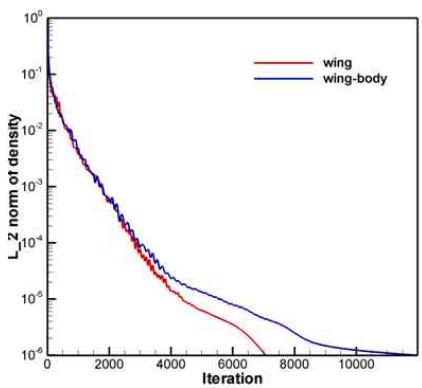

(b) Grid test

Fig. 4. Euler grid generation of wing-body model 


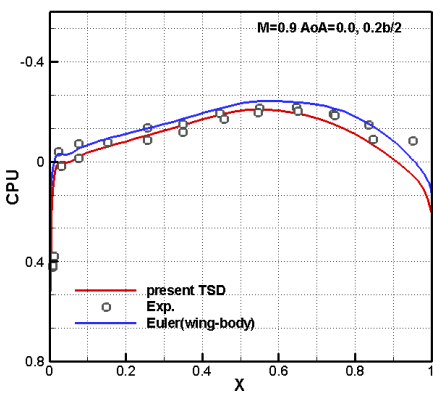

(a) $20 \%$ span

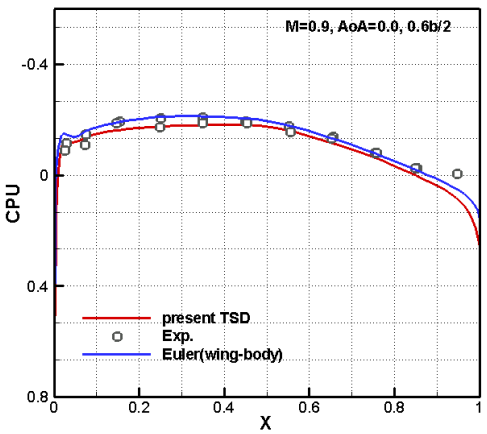

(c) $60 \%$ span

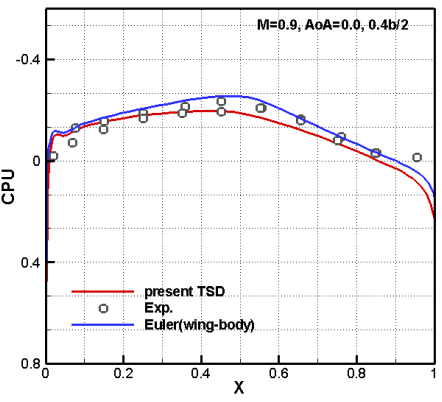

(b) $40 \%$ span

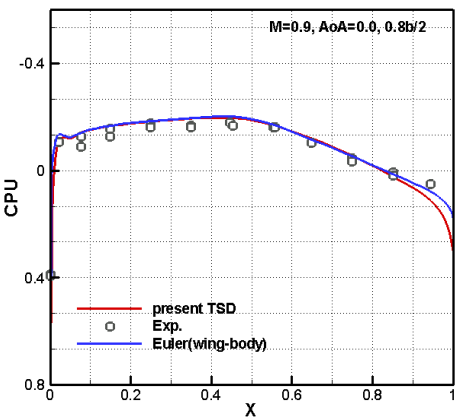

(d) $80 \%$ span

Fig. 5. Pressure coeff for Mach $=0.9$ and $A \circ A=0$ degree

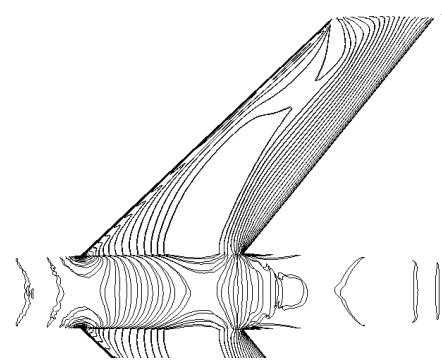

(a) wing-body model

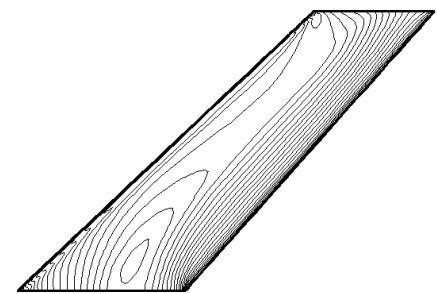

(b) wing model

Fig. 6. Pressure coeff. contour for Mach $=0.9$ and $A o A=0$ degree

\section{Conclusions}

In this research, the influence of body effect is studied. The body effect is implemented by introducing MGM inverse design method to TSD aerodynamic system. The MGM inverse design method is derived and validated by using airfoil shape tracking examples. Also, Euler wing-body model and TSD clean wing model are compared. From the result. body implemented model shows higher compressibility characteristics. The perturbation of body dissapears as it approachs to wing tip. The body interferenced region is enlarged according to the growth of Mach number.

\section{Acknowledgement}

This work was supported by Defense Acquisition Program Administration and Agency for Defense Development under the contract UD070041AD and by the second stage of the Brain Korea 21 Project in 2008. Authors are grateful for their supports. 


\section{References}

1. Malone, J. B., Narramore, J. C., and Sanker, L. N., 1990, "Airfoil Design Method Using the Navier-Stokes Equation", Journal of Aircraft, Vol. 28, No. 3.

2. Soemarwoto, B. I., Labrujère, Th.E., Laban, M., and Yansyah H., 1980, "Inverse Aerodynamic Shape Design for Improved Wing Buffet-onset Performance", NLR-TP-2000-150.

3. Kim, D. H., 2000, "Transonic/Supersonic Flutter Analysis of Wings with Control Surface and External Store", $P h^{\prime} D$ Thesis, KAIST, Korea.

4. Lee, S. J., Lee, I., and Han, J. H., 2006, "Transonic Flutter Suppression of the 2-D Flap Wing with External Store using CFD-based Aeroservoelasticity ", KASA International Journal, Vol. 1, No. 2.

5. Donald, L. L. and Bruce B. E.,1951, Research Memorandum, RML51F07.

6. Sung, C. H., Park, S. H., and Kwon, J. H., 2001, "Multigrid Diagonalized ADI Mehod for Compressible Flows," AIAA Paper 2001-2556. 